\title{
Avaliação da produção científica de um programa de pós-graduação stricto sensu em Odontologia como ferramenta de gestão
}

\author{
Lívia Mund de Amorim*; Rita Azevedo Senna*, Fabiana Vargas-Ferreira**, Paulo Floriani Kramer*** \\ * Mestre em Odontologia, Universidade Luterana do Brasil \\ ** Professora Doutora, Faculdade de Odontologia, Universidade \\ Federal de Minas Gerais \\ *** Professor Doutor, Curso de Odontologia, Universidade \\ Luterana do Brasil
}

Recebido em 02/09/2019. Aprovado em 13/04/2020.

\begin{abstract}
RESUMO
Na universidade, o ambiente de produção, disseminação e socialização do conhecimento se consolida pela realização da pesquisa e publicação de seus resultados. O presente estudo transversal objetivou identificar o perfil das dissertações, teses e publicações em periódicos do Programa de Pós-graduação Stricto Sensu em Odontologia da Universidade Luterana do Brasil (PPGOdonto/ULBRA). Realizouse uma análise bibliométrica quantitativa e descritiva no período de 1997-2016. Os dados, coletados por meio de formulário específico, consistiram em ano de defesa, especialidade e delineamento do estudo. Realizou-se uma busca na Plataforma Lattes e base de dados PubMed a fim de verificar as publicações em periódicos decorrentes dos trabalhos defendidos; e as publicações foram classificadas de acordo com os critérios Qualis. Os dados foram analisados por meio de estatística descritiva. Foram identificadas 284 dissertações de mestrado e 50 teses de doutorado defendidas no referido período. As áreas de Endodontia, Dentística e Prótese Dentária foram as especialidades com o maior número de defesas. Do total de dissertações e teses analisadas, $62 \%$ geraram publicações científicas, sendo $41 \%$ em periódicos de estratos Qualis A1 e A2, especialmente nas áreas de Endodontia, Periodontia e Odontopediatria. Em relação à metodologia utilizada nos estudos, 43,7\% utilizaram delineamento experimental in vitro, seguido de estudos transversais $(23,4 \%)$ e de estudos em modelo animal $(11,1 \%)$. A autoavaliação dos programas de pós-graduação com foco na postura crítica em relação ao que está produzindo tem permitido a reorientação de linhas de pesquisa e da dinâmica de geração de conhecimento e inovações.
\end{abstract}

Descritores: Pesquisa em Odontologia. Bibliometria. Publicação Periódica.

\section{INTRODUÇÃO}

Os cursos de pós-graduação stricto sensu são responsáveis pela pesquisa e geração de novos conhecimentos e tecnologias. No Brasil, a área de Ciências da Saúde é a que apresenta o maior número de programas. Os dados da avaliação da 
Coordenação de Aperfeiçoamento de Pessoal de Nível Superior (CAPES) no quadriênio 2013-2016 revelam que houve um crescimento na área de Odontologia em todas as regiões do Brasil, contabilizando 77 programas acadêmicos e 24 programas profissionais. Ainda persiste, contudo, uma polarização destes programas nas regiões Sul e Sudeste ${ }^{1}$.

Ao se contextualizar a produção científica da Odontologia brasileira, observa-se que esta responde por $85,2 \%$ do que é produzido na América Latina e ocupa a segunda posição na produção intelectual mundial de artigos indexados na base Scopus ${ }^{1}$. No quadriênio 2013-2016 foram publicados 15.921 artigos completos, sendo 5.897 (37\%) nos estratos Qualis A1 ou A2. Houve incremento quando comparado ao período anterior, evidenciando claramente a maturidade científica e a consolidação da área da Odontologia no cenário nacional e internacional ${ }^{1}$.

O perfil da produção científica de um programa de pós-graduação em um período específico pode ser identificado a partir da bibliometria. Esta técnica, no contexto científicoacadêmico, surge como um auxílio estatístico que permite mapear e gerar diferentes indicadores de tratamento e gestão da informação do conhecimento $^{2}$. Além disso, identifica os temas de maior destaque e as inovações ocorridas ao longo do tempo e seu possível caminho para o desenvolvimento no futuro ${ }^{3}$.

Este estudo tem por objetivo identificar, por meio de uma análise bibliométrica, o perfil das dissertações, teses e respectivas publicações em periódicos do Programa de Pós-graduação Stricto Sensu em Odontologia da Universidade Luterana do Brasil (PPGOdonto/ULBRA). no período de 1997-2016.

\section{METODOLOGIA}

Este estudo transversal caracteriza-se como uma pesquisa bibliométrica com abordagem quantitativa precedida por uma análise estatística descritiva. O material investigado consistiu em dissertações e teses defendidas no PPGOdonto/ULBRA no período de 1997 a 2016, e respectivas publicações em periódicos até 2018.

Os dados foram coletados por meio de formulário específico contendo as seguintes informações: ano de defesa da dissertação ou tese, nome do estudante, nome do orientador, título, especialidade e delineamento do estudo. Os dados foram obtidos dos registros do PPGOdonto/ULBRA. Um pesquisador experiente realizou a leitura e coleta de dados dos resumos dos trabalhos. Em caso de dúvida, o trabalho foi consultado na íntegra. Realizou-se busca dos currículos dos estudantes e orientadores na Plataforma Lattes (Conselho Nacional de Desenvolvimento Científico e Tecnológico, CNPq) e, adicionalmente, na base de dados PubMed (nome do autor e descritores), a fim de verificar se as dissertações e teses resultaram em publicações em periódicos. Os delineamentos foram categorizados como revisão de literatura (sistemática ou narrativa), ensaio clínico randomizado, coorte, caso-controle, transversal, série de casos, quasi-experimental, experimental (modelo animal, in situ e in vitro), intervenção nãocontrolada, análise documental, bibliometria, abordagem qualitativa e estudo de validação $0^{4,5}$. As especialidades foram categorizadas como Endodontia, Dentística, Prótese Dentária, Periodontia, Cirurgia e Traumatologia Bucomaxilofacial, Odontopediatria, Ortodontia, Radiologia Odontológica e Imaginologia, Implantodontia e Estomatologia ${ }^{6}$. As publicações em periódicos foram classificadas, de acordo com os critérios Qualis CAPES, em A1, A2, B1, B2, B3, B4, B5, C e sem classificação ${ }^{7}$. Os dados foram tabulados com o auxílio do Software STATA ${ }^{\circledR}$, Versão 12.0 (Stata Corp., College Station, TX, USA), sendo analisados por meio de estatística descritiva. 


\section{RESULTADOS}

No período em estudo, 334 trabalhos (284 dissertações de mestrado e 50 teses de doutorado) foram defendidos no PPGOdonto/ULBRA. O gráfico 1 ilustra o número de defesas realizadas por ano. O maior número de defesas de dissertações (50/18\%) ocorreu em 2003. As defesas de teses iniciaram em 2008 e o maior número ocorreu em 2015 (12/24\%). No último quadriênio (2013-2016) foram defendidos 76 trabalhos: 44 dissertações e 32 teses.

O gráfico 2 apresenta $\mathrm{o}$ número de publicações em periódicos realizadas por ano. É possível identificar que as dissertações e teses resultaram em 207 artigos publicados, representando $62 \%$ dos trabalhos defendidos, sendo $58 \%$ das dissertações e $82 \%$ das teses. Observa-se que o maior número de publicações ocorreu em 2015 (18/9\%). No último quadriênio (2013-2016) foram publicados 52 artigos em periódicos.

$O$ gráfico 3 apresenta a distribuição das dissertações, teses e respectivas publicações, de acordo com a especialidade. A Endodontia foi a área que acumulou o maior número de defesas $(56 / 17 \%$ do total), seguida pela Dentística (51/15\%) e Prótese Dentária (48/14\%). Observa-se ainda que a Odontopediatria e a Radiologia Odontológica e Imaginologia foram as áreas com maior número de teses defendidas, representando $42 \%$ do total. Em relação às publicações, verifica-se que a Endodontia foi a especialidade com o maior número de publicações $(55 / 26 \%$ do total), seguida pela Periodontia (42/20\%) e Odontopediatria (32/16\%), sendo que das dissertações e teses destas áreas $98 \%$, $93 \%$ e $89 \%$, respectivamente, foram publicadas.

No gráfico 4 encontram-se os delineamentos metodológicos mais utilizados nas dissertações, teses e respectivas publicações. Dos 334 trabalhos defendidos, $146 \quad(43,7 \%)$ apresentavam delineamento experimental in vitro, 78 (23,4\%) transversal, $37(11,1 \%)$ experimental em modelo animal, 19 (5,7\%) ensaio clínico randomizado (ECR) e $14 \quad(4,2 \%)$ quasi-experimental, representando $88 \%$ dos delineamentos dos trabalhos defendidos no PPGOdonto/ULBRA. Ensaios clínicos randomizados e revisões sistemáticas, por sua vez, representam menos de $10 \%$ dos delineamentos utilizados.

O gráfico 5 apresenta a distribuição das publicações por especialidade e classificação Qualis/CAPES. Os periódicos nos estratos Qualis A1 e A2 representaram 41\% (86) das publicações no referido período. A Endodontia, Periodontia e Odontopediatria se destacaram com $64 \%$ do total de artigos publicados nos estratos Qualis A1 e A2.

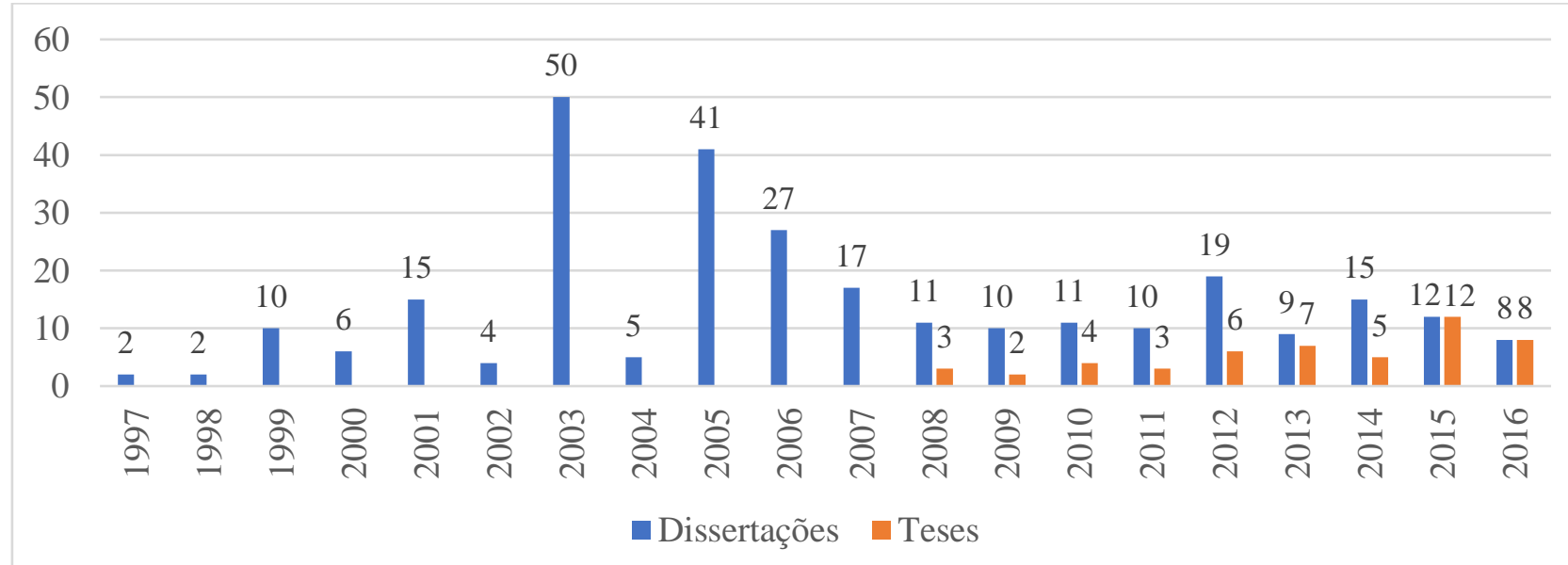

Gráfico 1. Distribuição das Dissertações e Teses do PPGOdonto/ULBRA (n=334), de acordo com o ano de defesa 


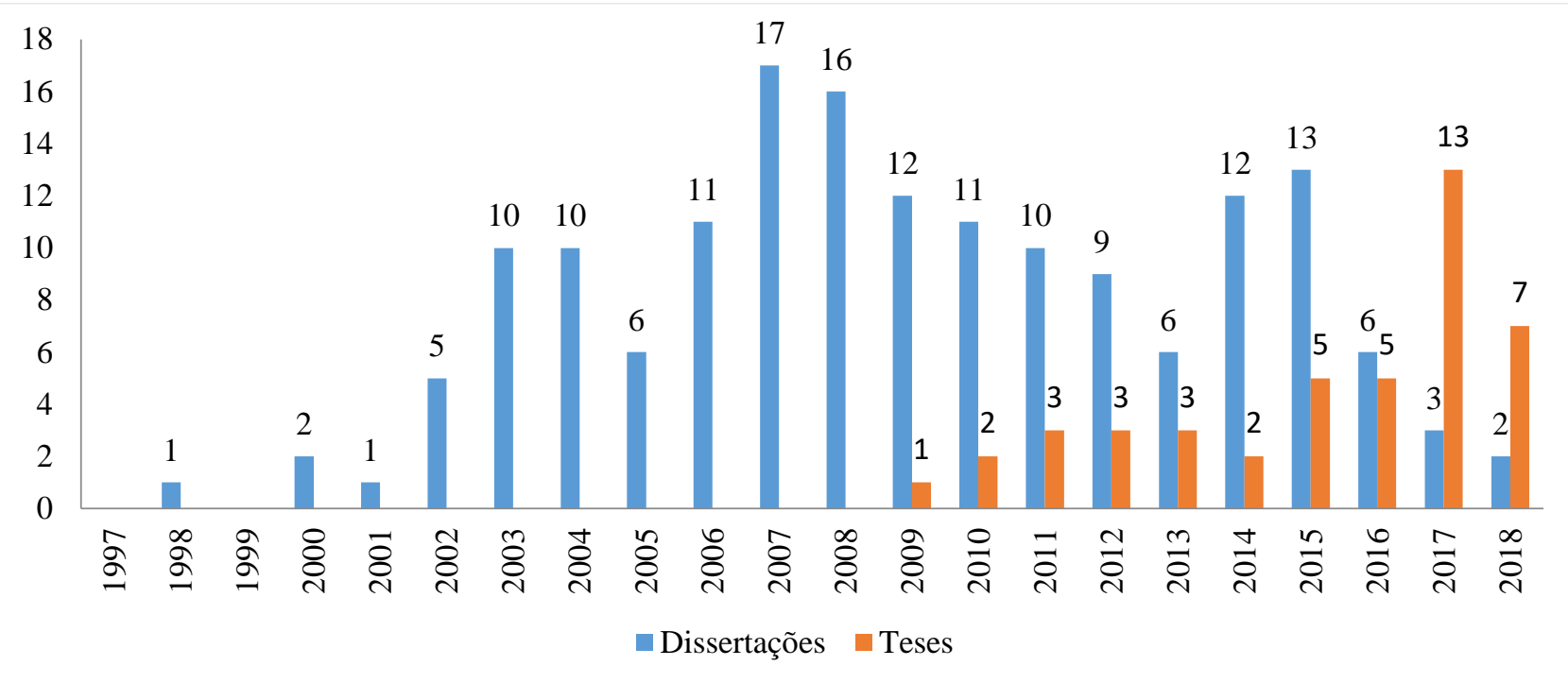

Gráfico 2. Distribuição das Publicações em Periódicos do PPGOdonto/ULBRA (n=207), de acordo com o ano de publicação

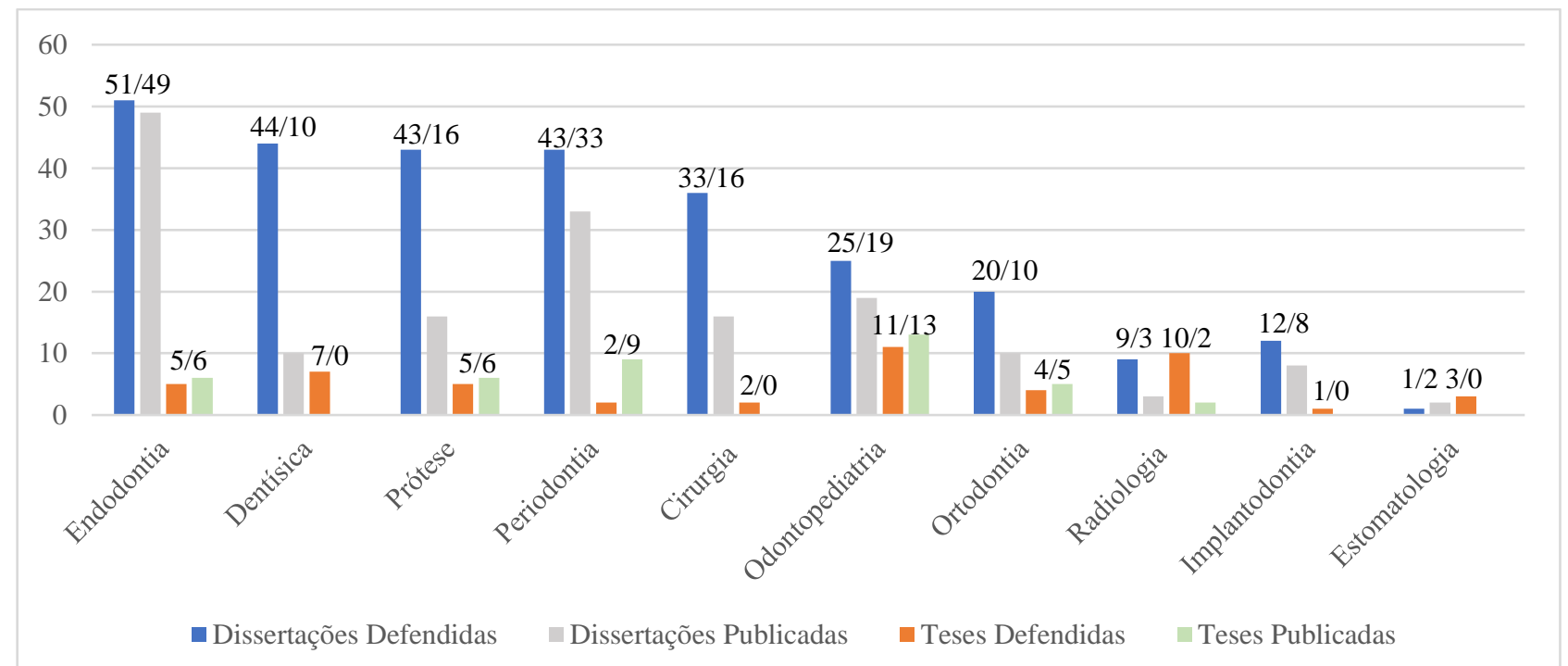

Gráfico 3. Distribuição das Dissertações $(n=284)$, Teses $(n=50)$ e respectivas Publicações em Periódicos $(\mathrm{n}=207)$ do PPGOdonto/ULBRA, de acordo com a especialidade 


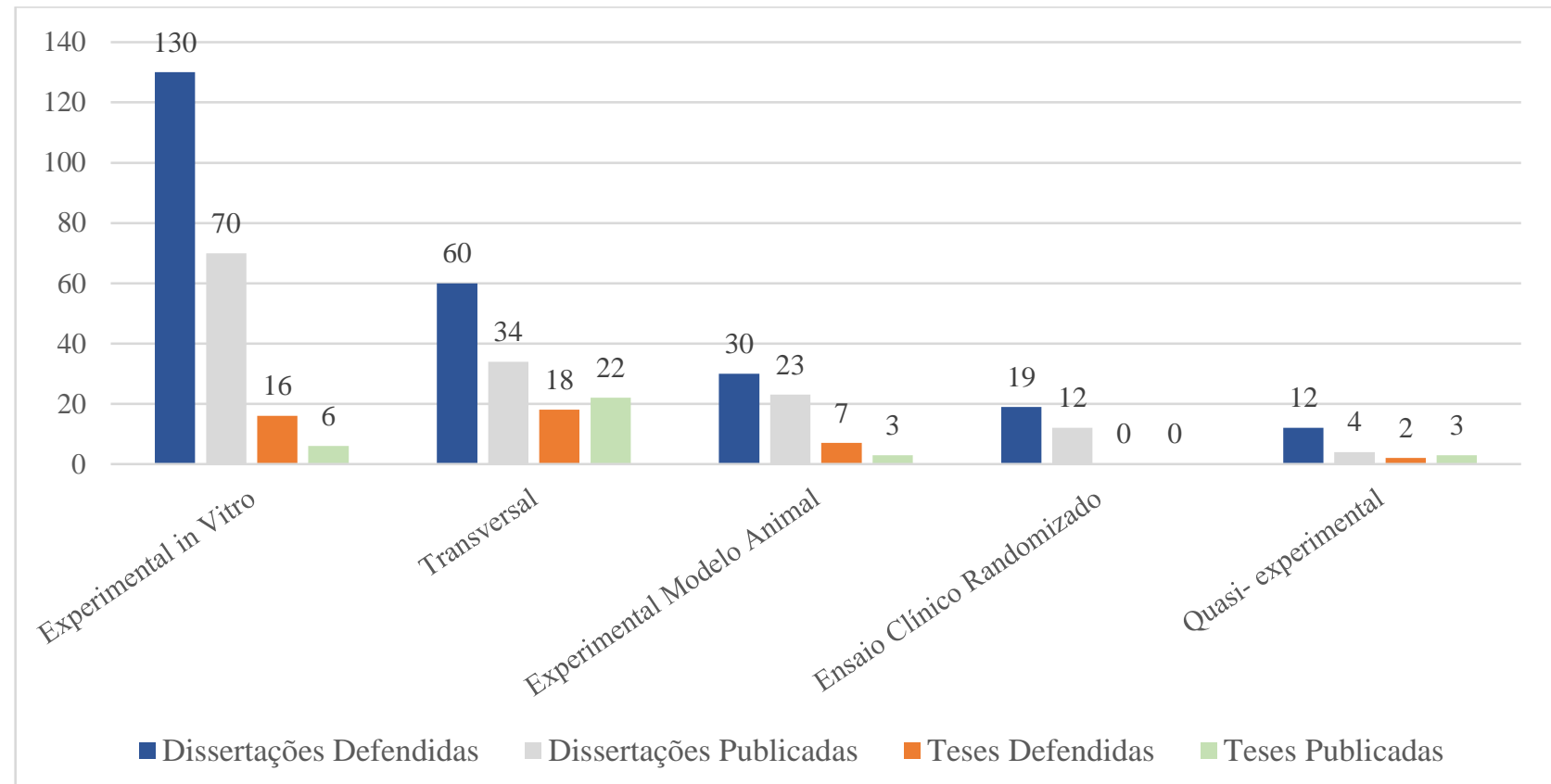

Gráfico 4. Distribuição das Dissertações $(n=284)$, Teses $(n=50)$ e respectivas Publicações em Periódicos $(\mathrm{n}=207)$ do PPGOdonto/ULBRA, de acordo com os delineamentos mais utilizados nos estudos

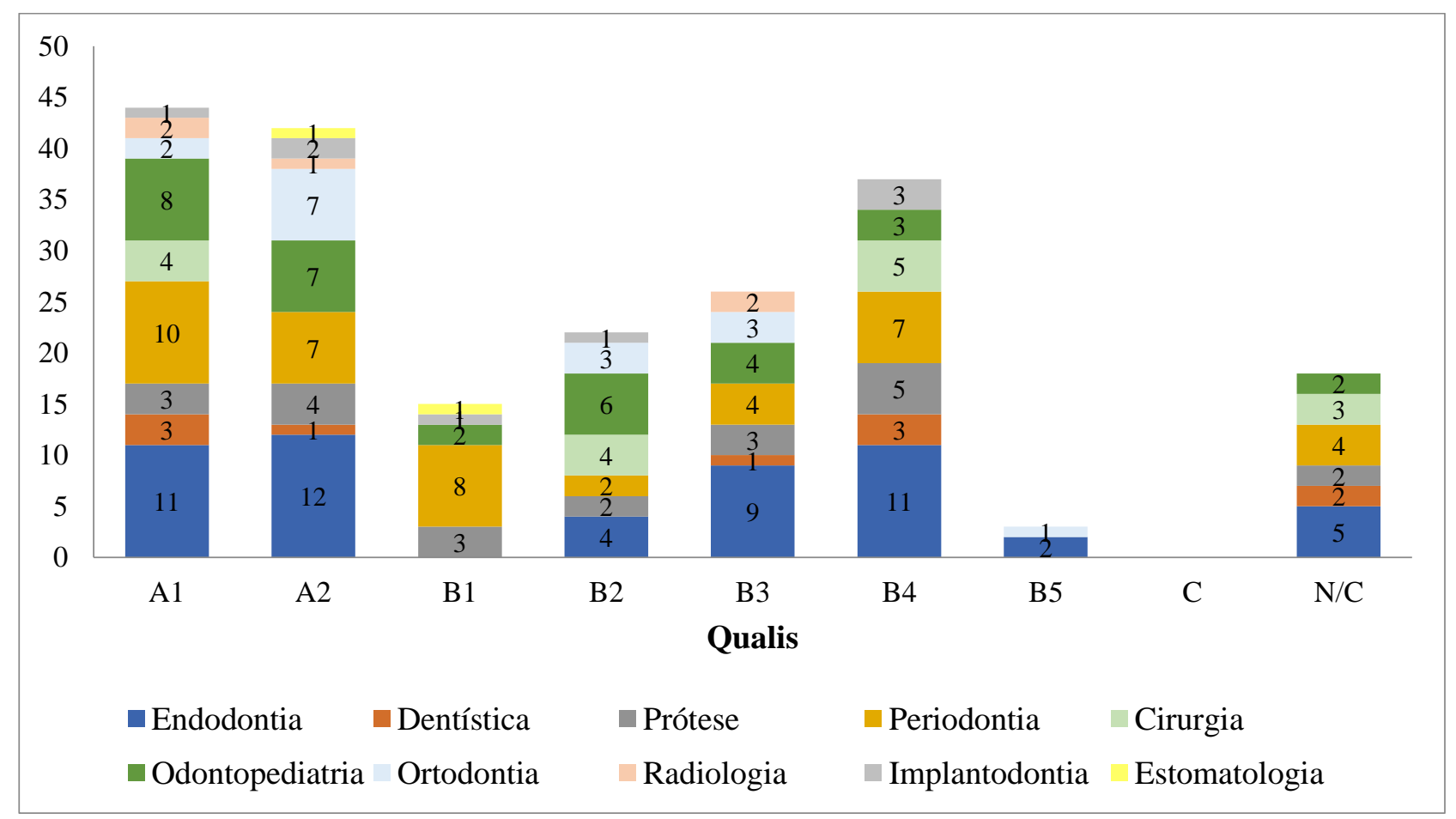

Gráfico 5. Distribuição das Publicações em Periódicos do PPGOdonto/ULBRA (n=207), de acordo com o Qualis/CAPES e a especialidade 


\section{DISCUSSÃO}

A política científica e tecnológica possibilita uma ampla variedade de recortes. Dentre suas temáticas, destacam-se os programas de pesquisa, instrumentos de financiamento, aspectos da legislação e a dinâmica de geração de conhecimento e de inovações. A conjuntura científica no Brasil revela o amadurecimento da postura crítica em relação ao que se está produzindo, especialmente quando se percebe a ampliação de estudos metacientíficos ou de avaliação dos modos de se fazer e compreender a ciência ${ }^{8,9}$

A Odontologia baseada em evidências é definida como o uso consciente, explícito e prudente da melhor evidência disponível para o cuidado. Pesquisas conduzidas em programas de pós-graduação, por sua vez, têm visibilidade por meio de revistas científicas de mérito, e a análise bibliométrica representa um importante instrumento no diagnóstico e monitoramento da divulgação do conhecimento científico ${ }^{2}$.

No presente estudo as especialidades de Endodontia, Dentística e Prótese Dentária acumularam o maior número de defesas de teses e dissertações. Essas áreas em questão se sobressaem, pois enfatizam domínios profissionalizantes do conhecimento odontológico $^{10}$. Além disso, há a influência do mercado na determinação da temática das pesquisas, reproduzindo o interesse direcionado para o complexo médico-farmacêutico-tecnológico demonstrado, principalmente, por pesquisas relacionadas à modernização e utilização dos materiais dentários ${ }^{11}$.

A divulgação de novos conhecimentos se dá, prioritariamente, por meio de periódicos - revistas especializadas em publicar informações originárias de diversos tipos de estudo na forma de artigos científicos $^{12}$. No presente estudo, foi possível observar significativa contribuição quanto à produção científica oriunda das dissertações e teses. Pode-se observar que $58 \%$ das dissertações e $82 \%$ das teses defendidas resultaram em publicações científicas. Martelli-Júnior et al. $(2007)^{13}$, mencionam que a produção científica odontológica brasileira tem aumentado, evidenciando a aculturação progressiva dos docentes de ensino superior sobre a necessidade de produzir conhecimento científico e publicar.

Dissertações e teses nas áreas de Endodontia, Periodontia e Odontopediatria foram responsáveis por $62 \%$ do total das publicações. Investimentos feitos por agência de fomentos (CAPES e CNPq) são direcionados para formação e absorção de recursos humanos e para projetos de pesquisa que contribuem para o aumento de produção de conhecimento e inovação para o país ${ }^{14}$. A não publicação desses trabalhos interrompe o ciclo que a agência de fomento está financiando, fazendo com que o recurso público seja desperdiçado. Há possibilidade de, a partir do perfil das publicações, direcionar as escolhas para temas de pesquisa, delineamentos metodológicos e projeções científicas e tecnológicas da pós-graduação na instituição.

Políticas adotadas e fortalecidas por agências de pesquisa estimulam os sistemas de avaliação e proporcionam parâmetros para evidenciar as meritocracias científicas $^{8}$. Níveis de evidência baseados no desenho e característica do estudo favorecem a análise da qualidade dos trabalhos publicados ${ }^{15}$. Verificou-se nos trabalhos defendidos uma predominância de delineamentos que apresentam baixo potencial para gerar evidências, destacando-se estudos do tipo experimental in vitro e experimentais em modelo animal. Esses achados estão de acordo com o perfil científico de trabalhos apresentados na Sociedade Brasileira de Pesquisa Odontológica (SBPqO), onde predominam estudos de cunho laboratorial ${ }^{16}$. Ensaios clínicos randomizados e revisões sistemáticas da literatura obtiveram uma taxa reduzida frente aos delineamentos anteriores. É 
importante salientar, contudo, que o aperfeiçoamento nos cuidados da saúde depende de resultados de trabalhos conduzidos e focados na investigação e sintetização de evidências relevantes e de qualidade ${ }^{17,18}$. Além de possuírem potencial de contribuição frente a definição de condutas clínicas e avaliar a cura de doenças, a sobrevivência de pacientes e a diminuição de sequelas, aumentam a chance de publicações em periódicos de maior fator de impacto ${ }^{19,20}$.

A classificação QUALIS representa a posição de referência que um periódico possui em relação à sua indexação em bancos de dados nacionais e internacionais, bem como o impacto dessa produção perante a comunidade científica ${ }^{21}$. No presente estudo observou-se que as publicações nos estratos Qualis A1 e A2 representaram 41\% das publicações realizadas no período avaliado, com destaque para as áreas de Endodontia, Periodontia e Odontopediatria.

A presente análise bibliométrica da produção científica do PPGOdonto/ULBRA nos permite avançar no diagnóstico do estado da arte do programa e em estratégias futuras para as diferentes especialidades. Áreas mais produtivas foram responsáveis por aproximadamente $2 / 3$ do total de publicações em periódicos. Ademais, foram responsáveis por aproximadamente $70 \%$ das publicações Qualis A1 e A2. São parâmetros importantes que podem auxiliar na gestão do programa, como por exemplo redefinir linhas e grupos de pesquisa e rediscutir delineamentos de estudos.

A indissociabilidade entre ensino e pesquisa na interface graduação/pós-graduação orienta a estrutura e a organização das instituições ensino superior desde o século passado; embora sempre tenha existido tensão entre o discurso historicamente disseminado e a realidade acadêmica. A universidade, contudo, tem avançado no processo, tendo em vista as tendências de valorizar a formação científica precocemente por meio de programas de iniciação científica, formação de grupos de pesquisa envolvendo docentes e estudantes de graduação e pósgraduação, e redefinição de linhas de pesquisa respondendo às necessidades dos projetos pedagógicos dos cursos. Essa interação entre os diferentes níveis tem permitido o intercâmbio de conhecimento, mobilidade acadêmica e desenvolvimento científico; contribuindo no sentido de consolidar uma educação superior de qualidade, pautada na autonomia do indivíduo/cidadão e conectada com as exigências da sociedade contemporânea.

As instituições de ensino superior, conscientes de seu compromisso e responsabilidade social, devem destacar-se distintamente de maneira integradora e coletiva, fortalecendo seus potenciais de reconhecimento e, destarte, na obtenção dos indicadores de excelência de visibilidade nacionais e internacionais. As perspectivas do processo de internacionalização dos programas de pós-graduação stricto sensu são uma realidade e vislumbram a importância e urgência no contexto de colaborações quanto à produção científica no cenário internacional, contribuindo para o aumento de citações. Além disso, intercâmbios de discentes e docentes mediante projetos colaborativos favorecem publicações conjuntas e participação ativa em eventos científicos internacionais ${ }^{22}$.

\section{CONCLUSÕES}

Com base nos resultados obtidos, conclui-se que no período de 1997 a 2016 foram defendidos 334 trabalhos no PPGOdonto/ULBRA e publicados 207 artigos em periódicos, representando $62 \%$ dos trabalhos defendidos. As áreas de Endodontia, Dentística e Prótese Dentária concentraram o maior número de defesas, enquanto a Endodontia, Periodontia e Odontopediatria foram as áreas com o maior número de publicações. Os delineamentos metodológicos do tipo experimental 
in vitro, transversal, experimental em modelo animal, ensaio clínico randomizado e quasiexperimental representaram $88 \%$ dos delineamentos utilizados nos trabalhos defendidos. Publicações Qualis A1 e A2 representaram 41\% das publicações do programa, sendo que aproximadamente $70 \%$ dos artigos publicados foram oriundos das áreas de Endodontia, Periodontia e Odontopediatria.

\section{ABSTRACT \\ Evaluation of the scientific production of a stricto sensu post-graduation program in Dentistry as a management tool}

At a university, the production, dissemination and socialization of knowledge are consolidated through research and the publication of results. The aim of the present cross-sectional study was to identify the characteristics of dissertations, theses of the Stricto Sensu postgraduate program in dentistry of the Lutheran University of Brazil and subsequent publication in journals. For such, a descriptive, quantitative, bibliometric analysis was performed of the period from 1997 to 2016. The data were collected using a specific form addressing year of defense, specialty and study design. Searches were performed in the Lattes Platform and PubMed database to verify publications in journals of the articles stemming from the work defended. The publications were classified using the Qualis criteria and the data were analyzed using descriptive statistics. A total of 284 master's dissertations and 50 theses were defended in the period analyzed. The specialties with the largest number of defenses were endodontics, restorative dentistry and dental prosthetics. Among the total number of dissertations and theses analyzed, $62 \%$ generated scientific publications and $41 \%$ were published in journals with Qualis A1 and A2 ratings, especially those in the fields of endodontics, periodontics and pediatric dentistry. Regarding the methods, the largest portion used an in vitro experimental design (43.7\%), followed by the cross-sectional design $(23.4 \%)$ and studies involving animal models $(11.1 \%)$. The selfevaluation of postgraduate programs with a focus on a critical stance with regards to what is being produced enables the reorientation of lines of research as well as the dynamics of knowledge generation and innovation.

Descriptors: Dental Research. Bibliometrics. Periodical.

\section{REFERENCIAS}

1. CAPES. Brasil. Ministério da Educação. Coordenação de Aperfeiçoamento de Pessoal de Nível Superior. Relatório Quadrienal Odontologia. [Internet]. 2017 [Acesso em 15 out. 2018] Disponível em: http://capes.gov.br/images/stories/download/a valiacao/relatoriosfinais-quadrienal-2017/20 122017-ODONTOLOGIA-quadrienal.pdf.

2. Bervian J, Bruch CM, Rodrigues PH, Poletto VC, Kramer PK. Análise bibliométrica da produção científica da Revista da Faculdade de Odontologia da Universidade de Passo Fundo. RFO, Passo Fundo. 2011;16(3):244-51.

3. Leite BDF, Menêzes TB, Noro LRA. Análise bibliométrica de Trabalhos de Conclusão de um Curso de Odontologia no Nordeste brasileiro. Rev ABENO. 2015;15(3):16-25.

4. Estrela C. Metodologia Científica: ensino e pesquisa em odontologia. São Paulo: Artes Médicas; 2001. p. 121-43.

5. Hochman B, Nahas FX, Filho RSO, Ferreira LM. Desenhos de pesquisa. Acta Cir Bras. 2005;20(2):11-32.

6. Conselho Federal de Odontologia. Consolidação das normas para procedimentos nos conselhos de odontologia. [Internet]. 2012 [Acesso em 15 out. 2018] Disponível em: $\underline{\mathrm{http}: / /}$ transparencia.cfo.org.br/wp-content/uploads /2018/03/consolidacao.pdf.

7. CAPES. Brasil. Ministério da Educação. Coordenação de Aperfeiçoamento de Pessoal de Nível Superior. Relatório Final 2016. [Internet]. 2017 [Acesso em 15 out. 2018] Disponível em: http://www.capes.gov.br/ 
images/stories/download/relatorios/231117Relatorio-PNPG Final-2016-CS.pdf.

8. Bufrem LS, Silveira M, Freitas JL. Políticas de ciência, tecnologia e inovação no Brasil: panorama histórico e contemporâneo. Revista P2P \& Inovação. 2018; 5(1):6-25.

9. Dias, RB. O que é a política científica e tecnológica. Sociologias. 2011; 13(28): 316-44.

10. Cavalcante R, Barbosa D, Bonan P. Perfil dos pesquisadores da área de Odontologia no Conselho Nacional de Desenvolvimento Científico e tecnológico (CNPq). Rev Brasil Epidemiol. 2008; 11(1): 106-13.

11. Marin F, Rebello M, Mello ALSF, Finkler M. Ética e Bioética como temas de pesquisa em Odontologia: uma análise bibliométrica dos trabalhos apresentados nas reuniões da $\mathrm{SBPqO}$. Rev ABENO. 2016; 16(4): 51-60.

12. Oliveira GJ, Oliveira ES, Leles CR. Tipos de delineamento de pesquisa de estudos publicados em periódicos odontológicos brasileiros. Rev Odonto Cienc. 2007; 22(55):42-7.

13. Martelli-Júnior H, Vieira Júnior JR, Domingos MA, Barbosa DR, Brito-Júnior M, Bonan PR. Pesquisa Odontológica no Estado de Minas Gerais-Brasil: Análise retrospectiva de 1986 a 2006. Arq. Odontol. 2007; 43(1): 23-9.

14. Oliveira Filho RS. Fomento à publicação científica e proteção do conhecimento científico. Acta Cir. Bras. 2005;20(2):35-9.

15. Richards D. Which journals should you read to keep up to date? Evid Based Dent. 1998; 1:22-5.

16. Pontes KT, Silva EL, Macêdo Filho RA, silva DR, Lima FJ. Estudo bibliométrico da produção científica em endodontia. Arch Health Invest. 2017;6(9):435-8.
17. dos Santos JGRP, Barbosa FT, Fraga TS. A qualidade dos ensaios clínicos randomizados publicados no Jornal Vascular Brasileiro. J Vasc Bras. 2011; 10(1):9-16.

18. Galvão TF, Pereira MG. Revisões sistemáticas da literatura: passos para sua elaboração. Epidemiol Serv Saúde. 2014;23(1):183-4.

19. Al-Namankany AA, Ashley P, Moles DR, Parekh, S. Assessment of the quality of reporting of randomized clinical trials in paediatric dentistry journals. Int $\mathrm{J}$ Paediatr Dent. 2009;19(5): 318-24.

20. Menegaz AM, Silva AER, Cascaes AM. Intervenções educativas em serviços de saúde e saúde bucal: revisão sistemática. Rev Saude Publica. 2018;52(1): 1-14.

21. Gomes D, Agnoletto IG, de Souza, ML, Spiger V, Jakymiu, JRG, da Cunha Fugii E, et al. A produção científica da Odontologia e a Agenda Nacional de Prioridades de Pesquisa em Saúde. Rev ABENO. 2017;17(2)11-21.

22. CAPES. Brasil. Ministério da Educação. Coordenação de Aperfeiçoamento de Pessoal de Nível Superior. Documento de Área 18 - Odontologia. [Internet] 2019. [Acesso em 02 mar. 2019] Disponível em: http://capes.gov.br/images/Documento de \%C3\%A1rea 2019/documento de area od onto.pdf.

\section{Correspondência para:}

Lívia Mund de Amorim

e-mail: liviamamorim@hotmail.com

Av. Farroupilha, 8001 - São José 92425-900 Canoas/RS 